\section{Effects of NM-3 on lymphatic vessel density and vascular endothelial growth factor of colon cancer in orthotopic implantation model of a severe combined immune deficiency mice}

\author{
Jin-Shui Zhu, Zhi-Peng Xu, \\ Jian- Lian Chen, Qiang Zhang, Qun Sun \\ Department of Gastroenterology, Sixth \\ People's Hospital Affiliated to Shanghai \\ Jiaotong University, Shanghai, China
}

\section{Abstract \\ The molecular mechanisms involved colon} cancer tumorigenesis and development of colon cancer remain unclear. The aim of this study is to explore the inhibitive effects of NM3 on lymphatic vessel density and vascular endothelial growth factor of micrometastatic lesion of orthotopic implantated colon cancer in the severe combined immune deficiency (SCID) nude mice. Human colon cancer SW1116 cells were orthotopically implantated into the colon of the nude mice. Twenty-eight SCID nude mice were randomly divided into four groups (7 mice for each group) after one week feeding and then the nude mice were treated with carboplatin and NM-3 via intraperitoneal injection twice a week for 8 weeks. The mice were sacrificed after 8 weeks and the vascular endothelial growth factor-C (VEGF-C), VEGF-D, VEGF-R-3 and lymphatic vessel density (LVD) were analyzed using immunohistochemistry staining assay. LVD in NM-3 treated mice was significantly lower than that of control (normal saline treated) mice. The expression of VEGF-C, VEGF-D, and VEGF-R-3 and the expression of mRNA of VEGF-C, VEGF-D, and VEGF-R-3 in NM-3 treated mice were significantly lower than that of control mice. The NM-3 inhibited the growth of colon cancer in the SCID mice of orthotopic implantatation model, and this effect may be related to the inhibitive effects of NM-3 on the lymphangiogenesis and vascular endothelial growth factor in colon cancer. NM-3 and carboplatin played a synergistic role in inhibiting lymphangiogenesis of human colon cancer in SCID nude mice and the further investigation of molecular mechanisms involved in colon cancer metastasis will provide an important evidence for understanding of lymphangiogenesis of human colon cancer.

\section{Introduction}

Colon cancer is one of the most common gastrointestinal malignant tumors and it is one of the leading causes of cancer-related deaths worldwide. In the past five years, it is reported that the incidence of colon cancer in Asia is increased and the prognosis of colon cancer is related to the potential metastasis and lymphangiogenesis of the tumor. The prognosis of advanced colon cancer is very poor. Until now, the molecular mechanisms involved colon cancer tumorigenesis remains unclear.

Because of the lack of specific lymphatic endothelial cells (LEC) markers, our previous studies using LMD and ${ }^{27}$-based RNA amplification and cDNA microarray could not discriminate between lymphatic and blood vessels. ${ }^{2}$ Recently, some LEC specific markers (VEGF-C, VEGF-D, and VEGF-R-3) were discovered. NM3 is a novel angiogenesis inhibitor isolated from a culture filtrate of streptoverticillium eurocidicum. Previous studies have revealed that NM-3 could inhibit the expression of VEGF family and have direct inhibitory effects on vessel endothelial cells of colon cancer. It is also showed that NM-3 combined with carboplatin can suppressed the growth of human colon cancer SW1116 cells xenografted or orthotopic implanted in the nude mice. ${ }^{3}$ These results suggested that NM-3 combined with carboplatin could not only increase anticancer effect and induce the apoptosis of human colon cancer cells but also aggravate toxicity to the cancer cells. VEGF-R-3 is one of the specific markers of lymphatic vessels in tumors. Based on this evidence, we investigated whether NM-3 could inhibit lymphangiogenesis in SCID nude mice that orthotopically implanted human colon cancer from SW1115 cells. LVD, subtypes of VEGF, VEGF-C, VEGF-D, VEGF-R-3 and their mRNA were studied using podoplanin and FQ-PCR assays.

\section{Materials and Methods}

\section{Chemicals}

VEGF-C, VEGF-D, and podoplanin polyclonal antibodies were purchased from Santa Cruz Biotechnology, Inc. (USA). VEGF-R-3 polyclonal antibody was obtained from AMS Biotechnology (Europe). Colon cancer SW116 cell line and NM-3 were kindly provided by Professor Robert (New York University, USA).

\section{Animal model}

Male severe combined immune deficiency (SCID) nude mice were obtained from Shanghai Experimental Animal Center of
Correspondence: Jin-Shui Zhu, Department of Gastroenterology, Sixth People's Hospital Affiliated to Shanghai Jiaotong University, Shanghai 200233, China.

E-mail: zhujs1803@hotmail.com

Key words: Colon cancer, NM-3, carboplatin, lymphangiogenesis, FQ-PCR, podoplanin, severe combined immune deficiency.

This work was supported by the Natural Science Foundation of Shanghai, No. 02ZB14072.

Received for publication: 30 March 2010.

Revision received: 18 May 2010.

Accepted for publication: 8 October 2010.

This work is licensed under a Creative Commons Attribution 3.0 License (by-nc 3.0).

(C) Copyright J-S Zhu et al., 2010

Licensee PAGEPress, Italy

Gastroenterology Insights 2010; 2:e12

doi:10.4081/gi.2010.e12

Chinese Academy of Sciences (SPF, NO.SCXK033). Six to seven week-old and body weight of 20-25 g mice were used. Human colon cancer SW1116 (a poorly differentiated adenocarcinoma) cell line was originally derived from a primary tumor and maintained by passage in the subcutis of nude mice. Animal models were set up via orthotopic implantation of histologically intact tissue of human colon carcinoma. The nude mice were anesthetized with $4.3 \%$ trichloraldehyde hydrate. An incision was made through the left upper abdominal pararectal line. Then the peritoneal cavity was carefully exposed and a part of the serosa membrane in the middle of the greater curvature of the stomach was mechanically injured using scissors. A tumor piece was fixed on each injured site of the serosal surface. The stomach was returned to the peritoneal cavity, and then the abdominal wall and skin were closed. After 7 days, the mice were randomly separated into four groups with seven mice per group. Carboplatin (5 mg/kg), NM-3 (10 mg/kg), carboplatin (5 $\mathrm{mg} / \mathrm{kg}$ ) combined with NM-3 (10 mg/kg), or normal saline (control group) were intraperitoneally injected into the each mice group respectively, twice a week. Eight weeks later, the mice were sacrificed by cervical dislocation. Tumors were resected aseptically. Necrotic tissues were cut off and the remaining healthy tumor tissues were minced into pieces (about $2 \times 2 \mathrm{~mm}$ in diameter) using scissors and were kept in Hank's balanced salt solution. Each tumor piece was weighed and adjusted to $50 \mathrm{mg}$, and then the tumor samples were immediately frozen in liquid nitrogen for later use. 


\section{Immunohistochemistry staining} assay

Immunohistochemical staining of podoplanin, VEGF-C, VEGF-D and VEGF-R-3 was performed using the streptavidin-peroxidase technique. Paraffin embedded tissues were cut into $4 \mu \mathrm{m}$ thick sections and placed on saline coated slides. After deparaffinization, antigen retrieval was performed by immersing the sections in $10 \mathrm{mmol} / \mathrm{L}$ citrate buffer $(\mathrm{pH} 6.0)$ and heated twice in a microwave oven $\left(95^{\circ} \mathrm{C}\right)$ for 5 minutes each time. Endogenous peroxidase activity was blocked by incubation with $1 \%$ hydrogen peroxide in distilled water for 10 min. Tissue sections were incubated with normal donkey serum for $20 \mathrm{~min}$ at room temperature and then incubated with polyclonal antibodies against LYVE-1, Prox-1, VEGF-C, VEGF$\mathrm{D}$ and podoplanin in normal donkey serum for $2 \mathrm{~h}$ at room temperature. Bound antibodies were detected with secondary antibody and streptavidin-peroxidase complex, using diaminobenzi-dine tetrahvdrochloride as the substrate. The sections were counterstained with hematoxylin.

\section{Evaluation of immunohistochemical staining \\ IHC-image analysis was performed using} IMS Cell Analysis System (Shanghai Shengteng Ltd. China). Every section was analyzed in at least 3 different visual fields $(\times 250,2.96 \mathrm{~V}$, $2.51 \mathrm{~A})$ to assay positive areas, positive percentage and optical density. The expression value was calculated as $0.095 \times$ average of three values.

\section{Lymphatic vessel density counting}

After scanning the stained sections of podoplanin at low magnification $(\times 100)$, five areas of carcinoma with the greatest number of distinctly highlighted intratumoral lymphatic foci (hot spots) were selected and vessels were counted in a representative high magnification $(\times 200)$ field in each of these five areas. Single immunoreactive endothelial cells, or endothelial cell clusters separated from other microvessels were counted as individual microvessels. Endothelial staining in large vessels with tunica media and non-specific staining of nonendothelial structures were disregarded in microvessel counts. Mean visual microvessel density for podoplanin was calculated using the average of five counts.

\section{Fluorescence-based quantitative polymerase chain reaction}

Amplification was carried out in a volume of $50 \mu \mathrm{L}$ mixture, containing $0.5 \mu \mathrm{L}$ of each primer $(25 \mu \mathrm{mol} / \mathrm{L}), 0.5 \mu \mathrm{L}$ dNTPs (10 $\mathrm{mmol} / \mathrm{L}), 1 \mathrm{U}$ of Taq DNA polymerase, $10 \mu \mathrm{L}$ of $5 \times$ PCR buffer (with $\mathrm{Mg}^{2+}, 25 \mathrm{mmol} / \mathrm{L}$ ), $5 \mu \mathrm{L}$ of
cDNA templates and $0.5 \mu \mathrm{L}$ fluorescent probe, and then made up to a volume of $50 \mu \mathrm{L}$ with deionized water. The primer sequence of VEGF-C was: sense 5'-GAATTCTCGGTGTGTGACA-3' and antisense: 5'-CTATCTCACAGCCTTCCTG-3'. The primer sequence of VEGF-D was: sense 5'-CCCA- TGAAGCCTTGTTTACCA3 ' and antisense: 5'-TGGAAGCG-GAACGGAAACT-3'. The primer sequence of VEGF-R-3 was: sense 5'-ACGGCCT-TGGTGAGTGGC-3' and antisense: 5'-CGTTTGACTCCTCCGTGATGATG-3'. The FQ-PCR amplification was accomplished as the following: an initial denaturation at $93^{\circ} \mathrm{C}$ for 2 min was followed by 10 cycles of denaturation at $93^{\circ} \mathrm{C}$ for 45 sec, annealing at $55^{\circ} \mathrm{C}$ for $60 \mathrm{sec}$, and extension at $93^{\circ} \mathrm{C}$ for $45 \mathrm{sec}$ followed by 30 cycles. Finally, an additional extension was achieved for $1 \mathrm{~min}$ at $55^{\circ} \mathrm{C}$.

\section{Statistical analysis}

The results were expressed as mean \pm SD. Statistical analysis was performed using t test by the statistical software SAS version 8 . $\mathrm{P}<0.05$ was considered statistically significant.

Table 1. Effects of NM-3/carboplatin on lymphatic vessel density in different groups $($ mean $\pm S D)$.

$\begin{array}{lll}\text { Groulp } & \text { N } & \text { LVD }(\%) \\ \text { Control } & 7 & 7.35 \pm 0.55 \\ \text { NM-3 } & 7 & 4.72 \pm 0.50^{\mathrm{a}} \\ \text { Carboplatin } & 7 & 6.34 \pm 0.49 \\ \text { NM-3 plus carboplatin } & 7 & 2.17 \pm 0.13^{\mathrm{b}}\end{array}$

$\mathrm{aP}<0.05, \mathrm{bP}<0.01$ vs control group.

Table 2. VEGF-C, VEGF-D, VEGF-R-3 levels of orthotopic implantated tumor in different groups $($ mean $\pm \mathrm{SD})$.

$\begin{array}{lcccc}\text { Group } & \text { n } & \text { VEGF-C }\left(\mu m^{2}\right) & \text { VEGF-D }\left(\mu m^{2}\right) & \text { VEGF-R-3 }\left(\mu m^{2}\right) \\ \text { Control } & 7 & 2962.84 \pm 519.77 & 1882.15 \pm 359.38 & 2123.05 \pm 117.99 \\ \text { NM-3 } & 7 & 2106.01 \pm 437.11^{\mathrm{a}} & 1032.25 \pm 460.44^{\mathrm{b}} & 1222.05 \pm 470.80^{\mathrm{a}} \\ \text { Carboplatin } & 7 & 2835.14 \pm 509.12 & 1785.23 \pm 334.27 & 2104.02 \pm 114.37 \\ \text { NM-3 plus carboplatin } & 7 & 1136.07 \pm 216.09^{\mathrm{b}} & 837.25 \pm 160.28^{\mathrm{b}} & 760.13 \pm 206.16^{\mathrm{b}}\end{array}$

$\mathrm{a} P<0.05,{ }^{\mathrm{b}}<0.01$ vs control group.

Table 3. Expression of VEGF-C mRNA, VEGF-D mRNA, VEGF-R-3 mRNA of in different groups $($ mean $\pm \mathrm{SD})$

$\begin{array}{lcccc}\text { Group } & \text { n } & \text { VEGF-C mRNA } & \text { VEGF-D mRNA } & \text { VEGF-R-3 mRNA } \\ \text { Control } & 7 & 49.18 \pm 3.12 & 56.26 \pm 4.12 & 52.13 \pm 3.17 \\ \text { NM-3 } & 7 & 29.68 \pm 1.49^{\mathrm{a}} & 30.26 \pm 0.80^{\mathrm{a}} & 32.42 \pm 1.02^{\mathrm{a}} \\ \text { Carboplatin } & 7 & 41.10 \pm 2.91 & 52.17 \pm 3.89 & 48.35 \pm 2.97 \\ \text { NM-3 plus carboplatin } & 7 & 21.10 \pm 1.95^{\mathrm{b}} & 19.26 \pm 1.89^{\mathrm{b}} & 18.40 \pm 1.64^{\mathrm{b}}\end{array}$

aP $<0.05$, bP $<0.01$ vs control group.

\section{Results}

LVD count by staining of podoplanin, LVD in NM-3 group and NM-3 plus carboplatin group were significantly lower than that of control group (Table 1).

\section{The expression of VEGF subtypes in nude mice}

The expression of VEGF-C, VEGF-D, VEGF-R3 , VEGF-C mRNA, VEGF-D mRNA, and VEGF-R3 mRNA in the NM-3, and NM-3 in combination with carboplatin treated mice was significantly lower compared to that of the control group (Table 2,3), but there was no significant difference between carboplatin and control group (Table 2, 3) (Figure 1).

\section{Discussion}

NM-3 has special anti-tumor effect. NM-3 in combination with carbopltin may play an active synergistical role. However, human colon cancer treated by NM-3 in combination with carbopltin was not reported in the SCID nude mice. ${ }^{3}$

Up to date, angiogenesis and lymphangiogenesis have been reported to play an important role in metastasis of colon cancer. Some researchers found that VEGF-C over-expression tumor cells could promote metastasis of lymph node and increase tissue LVD. ${ }^{4-8}$ Inhibiting lymphangiogenesis and inducing apoptosis of colon cancer have become one of the new treatments of colon cancer. ${ }^{9-12}$ Specific markers, such as podoplanin, VEGF-C, VEGF-D,

\section{Lymphatic vessel density count using staining of podoplanin}


VEGFR-3 and the others, such as WeibelPalade body PAL-E D2-40 etc. of lymphatic endothelial cells (LEC) could be regarded as the treatment targets. As an inhibitor of angiogenesis, subsequent work has shown that NM3 exhibits direct cytotoxic effects on endothelial and carcinoma cells. Moreover, NM-3 has been found to potentiate the therapeutic effects of certain chemotherapeutic agents, including 5-fluorouracil, paclitaxel, and cyclophosphamide. Our previous study showed that NM-3 combined with carboplatin could suppress the growth of human colon cancer SW1116 cells in vivo and xenografted under abdominal skin of the nude mice, suggesting that NM-3 has complex effects which include inhibiting lymphangiogenesis, inducing apoptosis, increasing anticancer effect and aggravating toxicity in combination with carboplatin. Our previous studies showed the inhibitive effect of NM-3 on orthotopically implantated tumors from human gastric cancer (SGC7901) cell line in the SCID nude mice. ${ }^{1}$ But effects of NM-3 on orthotopic implantated human colon cancer were not reported. The VEGF family consists of seven members: VEGFA, VEGF-B, VEGF-C, VEGF-D, VEGF-E, VEGF-F, and placental growth factor (PIGF). VEGF-A acts through VEGFR-1 and -2 receptors, VEGF$\mathrm{C}$ and $-\mathrm{D}$ act through VEGFR-2 and VEGFR-3. Both of VEGF-C and VEGF-D are able to induce proliferation and migration of lymphatic endothelial cells in vitro. Some researchers studied the expression of VEGF-C and VEGF-D in human tumors and their possible connection to metastasis. The majority of such studies confirmed a positive correlation between growth factor expression and adverse oncological features. VEGF-R-3 was restricted to LECs in the adult. The importance of VEGF-R-3 for the development of the lymphatic vasculature has been shown recently. The correlation of VEGF-C/NEGF-R-3 axis to the lymphatic spread of tumors was documented in several experimental models and clinic pathological studies in a variety of human malignancies. Recently, it was reported that the serum VEGF-C levels might be a useful biomarker in determining the presence of lymph node metastasis and the serum VEGF-C levels correlate with VEGF-C tissue expression in patients with colon cancer. It was also showed that both VEGF-D and VEGFR-3 were independent prognostic biomarkers for identifying the poor prognosis in patients after resection of the colon adenocarcinomas. Podoplanin was originally found on the surface of rat glomerular epithelial cells (podocytes) and linked to flattening of foot processes (hence the name "podoplanin") that occurs in glomerular diseases. There are studies indicated that podoplanin is regulated by Prox-1. Studies also indicated that endithial cells expression podoplanin had the same structure as lymphatic endothelial cells, so it is a specif-
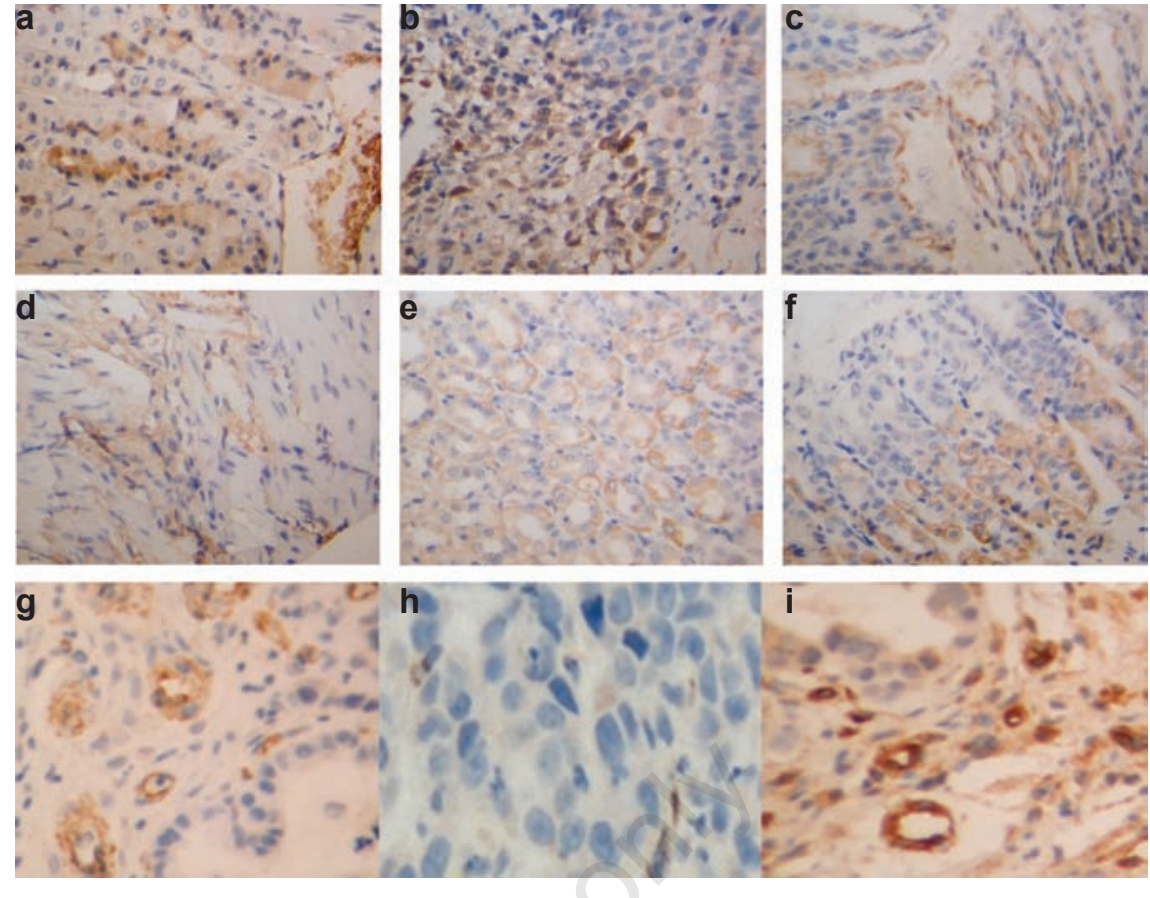

Figure 1. a) Expression of VEGF-C in control group. b) Expression of VEGF-D in control group. c) Expression of VEGF-R-3 in control group. d) Expression of VEGF-C in NM-3 group. e) Expression of VEGF-D in NM-3 group. $f$ ) Expression of VEGF-R-3 in NM-3 group. g) Expression of VEGF-C in NM-3 plus carboplatin group. h) Expression of VEGF-D in NM-3 plus carboplatin group. i) Expression of VEGF-R-3 in NM-3 plus carboplatin group.

ic marker of LECs. Proxl was also showed a general marker and tumor-associated lymphatic vasculature. In addition, it was also determined that Prox1, VEGF-R-3 and LYVE-1 were expressed similarly in lymphatic endothelial cells of normal adult and tumor tissues. In this study, we found that the expression of VEGF-C, VEGF-D, VEGF-R-3 and their mRNAs were decreased significantly after treatment of NM3 or NM-3 combined with carboplatin compared with those of control group, and the expression level of prox-1 was significantly different among the NM-3, NM-3 combined with carboplatin and carboplatin groups. The calculating of LVD also showed to be lower than control group after NM-3 or NM-3 and carboplatin treatment, which indicated that NM-3 has the effect of inhibiting lymphangiogenesis but carbopltin has not this function. These results also showed that combined drug use could play a stronger effect than the single drug use in inhibiting the growth of colon cancer cells. ${ }^{13-19}$ In our study, some results had no significant different between the two treatment groups which indicated that the major function of NM3 may inhibit lymphangiogenesis. ${ }^{20-26}$ Meanwhile, the quantity of models is so small and lack of NM-3 which made the results more valuable than theoretically. ${ }^{27-29}$

In conclusion, our studies suggested that NM-3 and carboplatin played a synergistic role in inhibiting lymphangiogenesis of human colon cancer in SCID nude mice and the further investigation of molecular mechanisms involved in advanced colon cancer will provide an important evidence for understanding of lymphangiogenesis of human colon cancer.

\section{References}

1. Zhu JS, Sheng B, Chen JL, et al. Molecular action mechanisms of NM-3 on human gastric cancer SGC-7901 cells in vivo or in vitro. World J Gastroenterol 2003;10:23669.

2. Zhu JS, Jiang XH, Cheng WX. Studies on peritoneal chemotherapy treatment of gastric cancer ascites and correlative effects. Hepato \& Gastroenterol 2000; 15:B108.

3. Wang $\mathrm{ZN}, \mathrm{Xu} \mathrm{HM}$, Jiang $\mathrm{L}$, et al. Expression of survivin aRNA in peritoneal lavage fluid from patients with Colon Cancer. Chin Med pJ 2004;117:1210-7.

4. Portera CA Jr, Berman RS, Ellis LM. Molecular determinants of colon cancer metastasis. Surg Oncol 1998;7:183-95.

5. Fidler IJ. Critical determinants of metastasis. Semin Cancer Biol 2002;12:89-96.

6. Emmert-Buck MR, Bonner RF, Smith PD, et al. Laser-capture microdissection. Science 1996;274:998-1001. 
7. BÖhm M,Wieland I, Schütze K, Rübben H. Microbeam moment: non-contact laser microdissection of membrane-mounted native tissue. Am J Pathol 1997;151:63-7.

8. Liotta L, Petricoin E. Molecular profiling of human cancer. Nat Rev 2000;1:48-56.

9. Wang L, Zhu JS, Song MQ, et al. Comparison of gene expression profiles between primary tumor and metastatic lesions in gastric cancer patients using laser microdissection and cDNA microarray. World J Gastroenterol 2006;12:694964.

10. Versteeg HH, Spek CA, Peppelenbosch MP, Richel DJ. Tissue factor and cancer metastasis: the role of intracellular and extracellular signaling pathways. Mol Med 2004; 10:6-11.

11. Kakinuma T, Hwang ST. Chemokines, chemokine receptors, and cancer metastasis.J Leukoc Biol 2006;79:639-51.

12 Cui Y, Ying Y, van Hasselt A, et al. OPCML is a broad tumor suppressor for multiple carcinomas and lymphomas with frequently epigenetic inactivation. PLoS ONE 2008;3:e2990.

13. Ye F, Zhang SF, Xie X, Lu WG. OPCML gene promoter methylation and gene expression in tumor and stroma cells of invasive cervical carcinoma. Cancer Invest 2008; 26:569-74.

14. Hayashida T, Ueda M, Aiura K, et al. Antiangiogenic effect of an insertional fusion protein of human basic fibroblast growth factor and ribonuclease-1. Protein Eng Des Sel 2005;18:321-7.

15. Mahajan NP, Whang YE, Mohler JL, Earp HS. Activated tyrosine kinase Ack1 pro- motes prostate tumorigenesis: role of Ack1 in polyubiquitination of tumor suppressor. Cancer Res 2005;65:10514-23.

16. Lynch G, Kohler S, Leser J, et al. The tyrosine kinase Yes regulates actin structure and secretion during pancreatic acinar cell damage in rats. Pflugers Arch 2004;447: $445-51$.

17. Ntougkos E, Rush R, Scott D, et al. The IgLON family in epithelial ovarian cancer: expression profiles and clinicopathologic correlates. Clin Cancer Res 2005;11:57648.

18. Reed JE, Dunn JR, du Plessis DG, et al. Expression of cellular adhesion molecule 'OPCML' is down-regulated in gliomas and other brain tumours. Neuropathol Appl Neurobiol 2007;33:77-85.

19. Sellar GC, Watt KP, Rabiasz GJ, et al. OPCML at 11q25 is epigenetically inactivated and has tumor-suppressor function in epithelial ovarian cancer. Nat Genet 2003;34:337-43.

20 Guo W, Shang F, Liu Q. Differential regulation of components of the ubiquitin-proteasome pathway during lens cell differentiation. Invest Ophthalmol Vis Sci 2004; 4:1194-201.

21. Wei W, Ayad NG, Wan Y. Degradation of the SCF component Skp2 in cell-cycle phase G1 by the anaphase-promoting complex. Nature 2004;6979:194-8.

22. Kudo Y, Kitajima S, Sato S. Transfection of p27(Kip 1) threonine residue 187 mutant type gene, which is not influenced by ubiquitin-mediated degradation, induces cell cycle arrest in oral squamous cell carcinoma cells. Oncology 2002;4:398-404.
23 Defatta RJ, De Benedetti A. Translational upregulation of yes accompanies eIF4Emediated oncogenic transformation. Int $\mathrm{J}$ Oncol 2000;23:1709-13.

24 Lawson C, Goupil S, Leclerc P. Increased Activity of the Human Sperm Tyrosine Kinase SRC by the cAMP-Dependent Pathway in the Presence of Calcium. Biol Reprod 2008;79:657-66.

25. Barraclough J, Hodgkinson C, Hogg A, et al. Increases in c-Yes expression level and activity promote motility but not proliferation of human colorectal carcinoma cells. Neoplasia 2007;9:745-54.

26. Psarras K, Ueda M, Tanabe M, et al. Targeting activated lymphocytes with an entirely human immunotoxin analogue: human pancreatic RNase1-human IL-2 fusion. Cytokine 2000;12:786-90.

27. Mahajan NP, Liu Y, Majumder S, et al. Activated Cdc42-associated kinase Ack1 promotes prostate cancer progression via androgen receptor tyrosine phosphorylation. Proc Natl Acad Sci 2007;104:8438-43.

28. Modzelewska K, Newman LP, Desai R, Keely PJ. Ack1 mediates Cdc42-dependent cell migration and signaling to p130Cas. J Biol Chem 2006;281:37527-35.

29. Castiglione F, Taddei A, Buccoliero AM, et al. TNM staging and T-cell receptor gamma expression in colon adenocarcinoma. Correlation with disease progression? Tumori 2008;94:384-8.

30 Iddings D, Bilchik A. The biologic significance of micrometastatic disease and sentinel lymph node technology on colorectal cancer. J Surg Oncol 2007;96:671-7. 